\title{
Nomogram for chemotherapy decision-making in patients with small triple-negative breast tumor: $A$ population-based study
}

\section{Xin-Yu Chen}

Fudan University Shanghai Cancer Center

\section{Yi-Lin Shao}

Fudan University Shanghai Cancer Center

\section{Xing Liu}

Fudan University School of Public Health

\section{Jian Zhang}

Fudan University Shanghai Cancer Center

\section{Wen-Xiang Zhi}

Fudan University Shanghai Cancer Center

Xi-Chun Hu

Fudan University Shanghai Cancer Center

Zhong-Hua Tao ( $\nabla$ drtaozhh@126.com )

Fudan University Shanghai Cancer Center https://orcid.org/0000-0003-3975-3803

\section{Research Article}

Keywords: triple-negative breast cancer, survival, nomogram, chemotherapy, STEPP

Posted Date: March 19th, 2021

DOl: https://doi.org/10.21203/rs.3.rs-337974/v1

License: (c) (i) This work is licensed under a Creative Commons Attribution 4.0 International License. Read Full License 


\section{Abstract}

Background: Small triple-negative breast cancer (TNBC) without lymph node invasion is considered less aggressive. This study aimed to develop nomograms for deciding on the appropriateness of chemotherapy for these patients.

Methods: The data of patients diagnosed with T1abNOMO TNBC between 2010 and 2016 were extracted from the Surveillance, Epidemiology, and End Results database. Clinicodemographic characteristics were integrated into nomograms for predicting overall survival (OS) and breast cancer-specific survival (BCSS). Nomogram performance was analyzed according to calibration and discrimination. The subpopulation treatment effect pattern plot (STEPP) was further applied to make individualized chemotherapeutic decisions.

Results: Overall, 3133 patients with T1abNOMO TNBC were evaluated; they were divided equally into the training and validation cohorts. Most tumors were grade III (62.6\%) and ductal carcinoma of no special type (88.8\%). The median follow-up time was 44.0 months, and 133 patients $(4.3 \%)$ died by the end of the follow-up. Eight independent prognostic factors, namely, age, race, marital status, tumor size, histology, grade, surgery, and chemotherapy, were identified and used in nomogram construction. The calibration curves showed satisfactory agreement between prediction and observation. The C-indices of the nomograms were significantly higher than those of traditional TNM staging ( 0.701 vs 0.503 for OS; 0.691 vs 0.506 for BCSS). The patients in the training cohort were stratified into two risk groups based on the OS nomogram score cutoff (213), and the groups showed a significant difference in survival, as was further confirmed in the validation cohort. The STEPPs of 5-year OS according to the nomogram score demonstrated distinct patterns of absolute chemotherapeutic effect, with low-risk patients achieving better survival regardless of chemotherapy. In high-risk patients, a significant and consistent chemotherapeutic benefit was observed at the average of $3.7 \%$ (range, $2.0 \%-4.9 \%$ ).

Conclusion: At a cutoff score of $\geq 213$, the nomogram accurately identified TNBC patients likely to receive a survival benefit from chemotherapy.

\section{Background}

About $15-20 \%$ of newly diagnosed breast cancer cases are triple-negative breast cancer (TNBC). TNBC is characterized by negative expressions of estrogen, progesterone, and human epidermal growth factor receptor 2 (HER2) receptors and no amplification of the HER2 gene.[1] Compared with other subtypes, TNBC is often associated with more visceral metastases,[2] higher risk of relapse,[3] and shorter progression-free survival (PFS) and overall survival (OS).[4-6] Small TNBC tumors often have good prognosis, especially $\mathrm{T} 1 \mathrm{abNOM0}$ tumors.[$[7,8]$ Hence, the absolute value of adjuvant chemotherapy for patients with T1abNOM0 remains uncertain.

The National Comprehensive Cancer Network Version 3.2020 includes several guidelines for chemotherapy decision-making. For tumors $\leq 0.5 \mathrm{~cm}(\mathrm{~T} 1 \mathrm{a})$, adjuvant chemotherapy is not necessary, 
while it may be generally beneficial for tumors measuring 0.6-1.0 $\mathrm{cm}(\mathrm{T} 1 \mathrm{~b})$. However, there are limited data for patients aged over 70 years and younger patients unable to tolerate commonly used treatment modalities.[9] The European Society of Medical Oncology recommends adjuvant chemotherapy for all PT1 stage tumors except those with certain low-risk histologic subtypes, such as secretory juvenile, apocrine, and adenoid cystic carcinomas.[10] The hesitation in recommending chemotherapy is because most studies focused on tumors $\geq 1.0 \mathrm{~cm}$ and/or with lymph node invasion, excluding patients with early stage tumors.[11-15]

Several retrospective studies have evaluated the benefit of adjuvant chemotherapy for TNBC pT1NOMO patients.[9, 16-19] A study of 264 patients with sub-centimetric TNBC tumors demonstrated improvement in distant-metastasis-free survival (DMFS) with chemotherapy within a median follow-up of 5.5 years. However, such DMFS benefit was not observed in another study of 284 patients with pT1abN0M0 TNBC over a 4-year median follow-up period. In a cohort of 354 consecutive Chinese TNBC patients, those with $\mathrm{T} 1 \mathrm{c}$ disease, but not those with $\mathrm{T} 1 \mathrm{~b}$ disease, had better recurrence-free survival (RFS) after adjuvant chemotherapy with a median follow-up of 3.8 years. A recent real-world study of 4366 newly diagnosed TNBC patients showed that adjuvant chemotherapy was associated with better breast cancer-specific survival (BCSS) and overall survival (OS) in patients with pT1N0M0 tumors compared with their non-chemotherapeutic counterparts, with the chemotherapeutic benefit most evident in pT1c. Apparently, these conflicting findings failed to specify the predictive factors or models to tailor adjuvant chemotherapy for sub-centimetric TNBC tumors.

The present study evaluated data on T1abNOMO TNBC obtained from a large population from the Surveillance, Epidemiology, and End Results (SEER) database. Nomograms predicting response to chemotherapy were created using multiple clinicopathological factors.

\section{Methods}

\section{Study design and population}

This study obtained data from the SEER database, which consists of data from 21 population-based cancer registries collected between 1975 and 2016. Data in the SEER database are representative of approximately $34.6 \%$ of the total US population. ASCII text version of the data was directly downloaded from the website (https://seer.cancer.gov).

We included female patients who had been pathologically diagnosed only with breast cancer in their lifetime. The inclusion criteria were age 18-90 years, TNBC subtype, tumor size $\leq 10 \mathrm{~mm}$ (T1a, b), NOM0 disease (American Joint Committee on Cancer [AJCC] 7th Edition), and tumor grade I-III. Patients diagnosed before 2010 were excluded due to the unavailability of data on their HER2 status. The other exclusion criteria were unknown age, histology, surgical treatment, chemotherapy use, or survival status. The patient inclusion flowchart is shown in Supplemental Fig. 1.

The need for informed consent was waived as data were collected from a publicly accessible source. 


\section{Nomogram construction}

To establish competing-risks nomograms and validate their usefulness, the patients were equally and randomly divided into the training and validation cohorts. Patients were classified into three major groups based on race/ethnicity: white, black, and others (including American Indian/Alaskan Native and Asian/Pacific Islanders). The "not married" status included single (never married), separated, divorced, unmarried or with domestic partner, and widowed.

\section{Validation and calibration of the nomograms}

The nomograms were subjected to 1000 bootstrap resamples for internal validation of the training cohort and of the validation cohort per se. The concordance index (C-index)[20] between predicted probability and response was used to assess the discrimination performance of the nomograms. The value of the $\mathrm{C}$ index theoretically ranges from 0.5 to 1.0 , with 0.5 indicating a random chance and 1.0 indicating a perfectly corrected discrimination. Calibration is the capability of a model to make unbiased estimates of outcome. Marginal estimate versus average predictive probability of the models was used to construct calibration curves. The prediction was expected to fall on a 45-degree diagonal line in a well-calibrated model.

\section{Survival stratification based on nomograms}

In addition to using C-indices to numerically compare the discrimination capability, we also evaluated the independent discrimination capability of the nomogram. Using $50 \%$ as cutoff, we divided the patients in the training cohort into two risk groups according to the OS prediction score. This cutoff score was then applied to the validation cohort, and Kaplan-Meier survival curves were generated.

\section{STEPP method}

To investigate absolute treatment effect patterns across the continuum of nomogram scores, the nonparametric sliding-window subpopulation treatment effect pattern plot (STEPP) methodology was applied.[21, 22] STEPP improves precision in a smoothing-by-binning manner by constructing overlapping subpopulations.[23]

\section{Statistical analysis}

OS was calculated from the date of diagnosis to the date of any-cause death or the last follow-up, whichever came first. BCSS was defined as the time interval from the date of diagnosis to the date of breast cancer death or the last follow-up. Deaths from other causes were regarded as competing risks, and such cases were censored at the date of the last follow-up. The cumulative incidence function was used to assess the probability of death. All univariate prognostic factors were compared using log-rank tests and entered into the multiple analysis via the Cox proportional hazards model. All significant variables, especially those associated with OS or with the cumulative incidences of breast cancer-related death, were adopted in the nomograms. The variables and the point assignment are listed in Supplemental table 1. All statistical analyses were performed using R version 3.6.1 (R Foundation for Statistical Computing. http://www.r-project.org). The R package rms was used for modeling and 
developing the nomograms.[24] All tests were two-sided, and $\mathrm{P}<0.05$ was considered statistically significant.

\section{Results}

\section{Patient characteristics}

In total, 3133 patients with pT1abNOMO TNBC were enrolled in this study. The clinicodemographic patient characteristics are shown in Table 1. The median age was 62 years (range, 24-90 years), and most patients were white $(75.9 \%$ ) and married (59.4\%). The median tumor size was $7 \mathrm{~mm}$, and most tumors were classified as grade III (62.6\%) and ductal carcinoma of no special type (88.8\%). Most patients underwent breast-conserving surgery (BCS, 71.5\%), and approximately half the cohort was administered chemotherapy (CT, 49.4\%) and radiotherapy (RAD, 59.0\%). The median follow-up time was 44.0 months (interquartile range, 26.0-62.0 months). By the end of the last follow-up, 133 patients (4.3\%) had died, of which $72(2.3 \%)$ died from breast cancer and 61 (2.0\%) died from other causes. 
Table 1

Patient characteristics $(n=3133)$

\section{Characteristics}

\section{Characteristics} Total population

$3133(100)$

Age at diagnosis, years

$18-39$
$40-49$
$50-59$
$60-69$
$70-79$
$80-90$
Race

White

Black

Others $^{\mathrm{a}}$

Unknown

Marital status at diagnosis

Not married ${ }^{b}$

Married

Unknown

CS tumor size $(\mathrm{mm})$

$[1,4]$

$[5,6]$

$[7,8]$
107 (3.4)

411 (13.1)

813 (25.9)

998 (31.9)

605 (19.3)

$199(6.4)$

2377 (75.9)

$518(16.5)$

$225(7.2)$

$13(0.4)$

1120 (35.7)

1862 (59.4)

$151(4.8)$

$629(20.1)$

643 (20.5)

841 (26.8)
$55(3.5)$

$200(12.8)$

405 (25.9)

499 (31.9)

294 (18.8)

$113(7.2)$

1198 (76.5)

251 (16.0)

113 (7.2)

$4(0.3)$

567 (36.2)

916 (58.5)

$83(5.3)$

317 (20.2)

319 (20.4)

419 (26.8)
52 (3.3)

211 (13.5)

408 (26.0)

499 (31.8)

311 (19.8)

86 (5.5)

1179 (75.2)

267 (17.0)

$112(7.1)$

9 (0.6)

553 (35.3)

$946(60.4)$

$68(4.3)$

312 (19.9)

324 (20.7)

422 (26.9)

${ }^{a}$ Other races include American Indian/AK Native, Asian/Pacific Islander.

${ }^{b}$ Not married includes single (never married), separated, divorced, unmarried or with a domestic partner, and widowed.

Data are presented as no. of patients (\%).

Abbreviations: NST, no special type; BCS, breast-conserving surgery; CPM, contralateral prophylactic mastectomy; CT, chemotherapy; RAD, radiation therapy 


\begin{tabular}{|c|c|c|c|}
\hline Characteristics & Total population & Training cohort & Validation cohort \\
\hline$[9,10]$ & $1020(32.6)$ & $511(32.6)$ & $509(32.5)$ \\
\hline \multicolumn{4}{|l|}{ Histology } \\
\hline Ductal carcinoma, NST & $2781(88.8)$ & $1386(88.5)$ & $1395(89.0)$ \\
\hline Other ductal and lobular neoplasms & $200(6.4)$ & $103(6.6)$ & $97(6.2)$ \\
\hline Others & $151(4.8)$ & $77(4.9)$ & $74(4.7)$ \\
\hline \multicolumn{4}{|l|}{ Grade } \\
\hline I & $184(5.9)$ & $99(6.3)$ & $85(5.4)$ \\
\hline II & $988(31.5)$ & $499(31.9)$ & 489 (31.2) \\
\hline III & $1961(62.6)$ & $968(61.8)$ & $993(63.4)$ \\
\hline \multicolumn{4}{|l|}{ Surgery of the primary site $\mathrm{e}^{\mathrm{e}}$} \\
\hline BCS & $2240(71.5)$ & $1121(71.6)$ & 1119 (71.4) \\
\hline CPM & $140(4.5)$ & $65(4.2)$ & $75(4.8)$ \\
\hline Other mastectomies & $753(24.0)$ & $380(24.3)$ & $373(23.8)$ \\
\hline \multicolumn{4}{|l|}{$\mathrm{CT}^{\mathrm{f}}$} \\
\hline No & $1585(50.6)$ & $822(52.5)$ & $763(48.7)$ \\
\hline Yes & $1548(49.4)$ & $744(47.5)$ & $804(51.3)$ \\
\hline \multicolumn{4}{|l|}{$\mathrm{RAD}^{\mathrm{g}}$} \\
\hline No & $1178(37.6)$ & $594(37.9)$ & $584(37.3)$ \\
\hline Yes & $1848(59.0)$ & $927(59.2)$ & $921(58.8)$ \\
\hline Unknown & $107(3.4)$ & $45(2.9)$ & $62(4.0)$ \\
\hline \multicolumn{4}{|c|}{ aOther races include American Indian/AK Native, Asian/Pacific Islander. } \\
\hline \multicolumn{4}{|c|}{$\begin{array}{l}\text { b Not married includes single (never married), separated, divorced, unmarried or with a domestic } \\
\text { partner, and widowed. }\end{array}$} \\
\hline \multicolumn{4}{|c|}{ Data are presented as no. of patients (\%). } \\
\hline $\begin{array}{l}\text { Abbreviations: NST, no special type; B } \\
\text { mastectomy; CT, chemotherapy; RAD, }\end{array}$ & $\begin{array}{l}\text { breast-conserving } \\
\text { iation therapy }\end{array}$ & gery; CPM, cont & teral prophylactic \\
\hline
\end{tabular}

\section{Overall survival}


The results of the univariate and multiple regression analyses in the training cohort based on a Cox proportional-hazards model are presented in Supplemental tables 2 and 3. Several variables, including age ( $\geq 70$ years, hazard ratio, $\mathrm{HR}=2.42, P<0.001$ ), surgery (mastectomies other than contralateral prophylactic mastectomy [CPM] HR $=2.00, P=0.008)$, and chemotherapy $(\mathrm{HR}=0.52, P=0.016)$, showed a significant correlation with OS. All significant factors were then included in multiple regression models, and age ( $\geq 70$ years $\mathrm{HR}=1.99, P=0.023$ ), marital status (married $\mathrm{HR}=0.64, P=0.049$ ), tumor size (every $1 \mathrm{~mm}$ increase: $\mathrm{HR}=1.14, P=0.024$ ), and surgery (mastectomies other than CPM HR=2.43, $P=0.026$ ) were identified as independent factors.

\section{Breast cancer-specific survival}

The results of univariate and multiple regression analyses of BCSS, along with estimates of probabilities of death resulting from breast cancer, are listed in Supplemental tables 2, 3, and 4. In univariate analysis, surgery (CPM: $\mathrm{HR}=3.62, P=0.041$; mastectomies other than $\mathrm{CPM:} \mathrm{HR}=3.07, P=0.001$ ) significantly correlated with BCSS. All factors were then subjected to multiple regression analysis. As expected, surgery remained significantly associated with BCSS (CPM: HR $=9.19, P=0.004$; mastectomies other than CPM: HR $=6.23, P<0.001)$.

Overall, the 3- and 5-year probabilities of death from breast cancer were .02 and .03 (Supplemental table 4). Consistent with regression analyses, surgery remained significantly associated with breast cancerspecific death; CPM had the highest incidence (5-year rate: 0.09), whereas breast-conserving surgery had the lowest incidence (5-year rate: 0.02).

\section{Nomogram validity}

Nomograms and their point assignment are presented in Fig. 1 and Supplemental table 1. Tumor size, histology, grade, and surgery were included in both nomograms, while age, marital status, and CT were applied solely for OS. In the OS nomogram, surgery (CPM: 78) exhibited the strongest influence, followed by age (66) and tumor size (11 for every $1 \mathrm{~mm}$ increase). In contrast, surgery remained the most significant factor influencing BCSS (CPM: 100). By adding all points and locating them on the bottom scales, we could calculate the estimated 3- and 5-year survival probabilities.

\section{Calibration and validation of the nomograms}

The calibration plots for the OS and BCSS nomograms in the training cohort and validation cohort are shown in Fig. 2 and Supplemental Fig. 2. Most mean predicted values and their 95\% confidence intervals covered the actual survival rate, further indicating concordance between the predicted and observed survival. Harrell's C-indices of the OS and BCSS nomograms were 0.702 [standard error (SE): 0.034] and 0.680 (SE: 0.045), respectively, in the validation cohort. These were similar to those of the training cohort: 0.701 (SE: 0.024) for OS and 0.691 (SE: 0.031) for BCSS. All C-indices were higher than 0.680, indicating that these two nomograms were satisfactorily accurate and suitable for prognosis prediction. In addition, these values significantly exceeded those of the traditional TNM staging system (OS: 0.503 (SE: 0.007), $P<0.001$; BCSS: 0.506 (SE: 0.011), $P<0.001$ ), as indicated by $99.7 \%$ of the patients having stage IA disease following the AJCC 7th Edition guidelines. 


\section{Application of the nomogram in identifying patients for chemotherapy}

To further assess the usefulness of the nomograms in identifying patients who will benefit from adjuvant chemotherapy, we calculated the OS prediction scores for patients in the training cohort. A $50 \%$ cutoff was used to divide them into two risk groups (low risk: $<213$; high risk: $\geq 213$ ). The risk score was significantly associated with survival in both the training cohort (OS: $\mathrm{HR}=0.34,95 \% \mathrm{Cl}=0.197-0.863, P$ $<0.001$; BCSS: $\mathrm{HR}=0.49,95 \% \mathrm{Cl}=0.24-0.99, P=0.043$ ) (Fig. $3 \mathrm{~A}$ and $\mathrm{B}$ ) and the validation cohort (OS: $\mathrm{HR}=0.29,95 \% \mathrm{Cl}=0.15-0.73, P<0.001 ; \mathrm{BCSS}$ : $\mathrm{HR}=0.47,95 \% \mathrm{Cl}=0.23-0.97, P=0.040$ ) (Figs. $3 \mathrm{C}$ and D). The high-risk group showed lower 5 -year OS (93.06\% vs $97.03 \%)$ and BCSS $(96.98 \%$ vs $98.70 \%)$ than the low-risk group.

The STEPPs of the 5-year OS according to the nomogram prediction score demonstrated distinct patterns of absolute chemotherapeutic effect (Fig. 4). In the low-risk patients, the 5-year OS was $98.0 \%$ independent of chemotherapy administration $(P=0.840$ in the training cohort and $P=0.900$ in the validation cohort, Fig. $4 \mathrm{~A}$ and $\mathrm{C}$ ). There was no apparent pattern across the prediction score spectrum, further indicating no clinically relevant improvement in 5-year OS with respect to chemotherapy. For highrisk patients who did not receive chemotherapy, the 5-year OS was $91.8 \%$. A consistent benefit of chemotherapy was evident across the continuum of prediction scores $(P<0.001$ in both the training and validation cohorts, Fig. 4B and D). With the average survival benefit being $3.7 \%$, the pattern of treatment effect according to prediction score distribution was also noticeable. Among subpopulations with the highest prediction score, the smallest absolute improvement of survival from chemotherapy was $2 \%$. Meanwhile, the chemotherapeutic absolute improvement reached $6.5 \%$ in subpopulations with approximately 250 points, and patients administered chemotherapy achieved a median 5-year OS of $95 \%$ (Fig. 4B). Similar chemotherapeutic effects in high-risk patients in the validation cohort were observed (Fig. 4D).

To confirm the effect of chemotherapy, we delineated OS and BCSS curves for each prediction score category in both cohorts (Fig. 5 and Supplemental Fig. 3). As expected, chemotherapy did not improve the OS and BCSS (HR $=1.07, P=0.911$ for both; all deaths were breast cancer specific) in low-risk patients (Fig. 5A and C). In contrast, chemotherapy significantly improved the OS ( $\mathrm{HR}=2.10,95 \% \mathrm{Cl}=1.08-8.21$, $P=0.032)$ and $\mathrm{BCSS}(\mathrm{HR}=2.96,95 \% \mathrm{Cl}=1.23-7.77, P=0.028)$ in high-risk patients (Fig. $5 \mathrm{~B}$ and $\mathrm{D})$. The association between chemotherapy and survival was further verified in the validation cohort (low-risk group: OS: $\mathrm{HR}=0.83,95 \% \mathrm{Cl}=0.28-2.49, P=0.743$; $\mathrm{BCSS}$ : $\mathrm{HR}=0.64,95 \% \mathrm{Cl}=0.17-2.49, P=0.521$ [Supplemental Fig. $3 \mathrm{~A}$ and $\mathrm{C}$; high-risk group: OS: $\mathrm{HR}=1.94,95 \% \mathrm{Cl}=1.05-3.62, P=0.040$; $\mathrm{BCSS}$ : $\mathrm{HR}=$ $2.71,95 \% \mathrm{Cl}=1.18-6.17, P=0.019$ [Supplemental Fig. 3B and $\mathrm{D}]$ ).

We further evaluated the relationship between $\mathrm{T} 1 \mathrm{a} / \mathrm{b}$ classification and nomogram score categorization. Although T1b patients have generally higher risks than T1a patients (Supplemental table $5, P<0.001$ ), approximately half of them (46.6\%) were classified into the low-risk category according to our nomogram score cutoff. This indicated that chemotherapy may not be necessary. However, a significant proportion of pT1a patients (42.0\%) were included in the high-risk subgroup. Thus, we subdivided the T1a and T1b patients according to the OS score cutoff (213) and analyzed survival differences associated with 
chemotherapy (Fig. 6, Supplemental Fig. 4). Patients with a low score did not have an OS benefit from chemotherapy regardless of tumor size $(\mathrm{T} 1 \mathrm{a}: \mathrm{HR}=1.28,95 \% \mathrm{Cl}=0.26-6.35, P=0.762 ; \mathrm{T} 1 \mathrm{HR}=0.766$, $95 \% \mathrm{Cl}=0.29-2.05, P=0.595$ [Figure $6 \mathrm{~A}$ and $\mathrm{C}$ ]). However, both $\mathrm{T} 1 \mathrm{a}$ and $\mathrm{T} 1 \mathrm{~b}$ high-risk patients showed a significant OS benefit from chemotherapy $(\mathrm{T} 1 \mathrm{a}: \mathrm{HR}=7.25,95 \% \mathrm{Cl}=1.01-53.57, P=0.050 ; \mathrm{T} 1 \mathrm{~b}: \mathrm{HR}=$ $1.94,95 \% \mathrm{Cl}=1.18-3.19, P=0.009$ [Figure 6B and D]). Similar trends were observed in the BCSS analyses (Supplemental Fig. 4). Collectively, nomogram score categorization, which combined tumor size with other clinicopathologic characteristics, could better identify patients who are likely to benefit from chemotherapy compared with the traditional $\mathrm{T} 1 \mathrm{a} / \mathrm{b}$ classification.

\section{Discussion}

Previous studies in untreated sub-centimetric TNBC patients reported a 5-year disease-free survival (DFS) of $80-90 \%,{ }^{15,16}$ suggesting that certain pT1ab TNBC patients have a high risk for recurrence and/or metastasis. This highlights the necessity of providing adjuvant chemotherapy for such patients. However, there is no clear guideline on selecting patients with pT1abNOMO TNBC who are likely to benefit from adjuvant chemotherapy. In this study, we established a novel clinical model to identify patients with PT1 abNOMO TNBC who have high risk of recurrence/metastasis. Those with a higher risk score had significantly worse OS and BCSS and derived a significant survival benefit from adjuvant chemotherapy.

Traditionally, the patients' clinicopathological characteristics (e.g., age; comorbidity; and tumor biology, including tumor grade, histological subtype, lymphatic vessel invasion [LVI], and stromal tumor-infiltrating lymphocytes), are used to evaluate the risk of recurrence/metastasis. The same characteristics are considered in the recommendation for adjuvant chemotherapy. However, it is difficult to accurately determine the risk for each patient. Although gene signature is an alternative tool, it currently cannot aid in determining the need for adjuvant chemotherapy for patients with pT1abNOMO TNBC because no prospective analysis of available gene assays (e.g., 21-gene assay (Oncotype Dx) and MammaPrint) has included such patients. $[25,26]$ Hence, it is difficult for physicians to identify the patients with a high metastatic potential based on clinical and biological data alone. Thus, STEPP analysis based on nomogram score was included in the present study. The nomograms showed satisfactory C-indices that significantly exceeded those of the traditional TNM staging system. This proves that in addition to tumor size, a combination of clinicopathologic factors including age, race, marital status, histology, grade, surgery, and chemotherapy could improve the prediction of outcomes for patients with T1abNOMO tumors. High-risk patients (i.e., those with nomogram scores above 213) were found to have a significant OS and BCSS benefit from chemotherapy.

Tumor size is an important prognostic factor in cancer. It was initially believed that large mammary tumors develop their metastatic capability through an accumulation of gene mutations. However, several studies have shown that certain small tumors also have strong metastatic capability, challenging this theory.[27, 28] The heterogeneity of cancer gives a reasonable explanation for such contradictory

Page $10 / 20$ 
findings. It is well-known that TNBC is a heterogeneous disease, exhibiting highly diverse and unsatisfactory outcomes. In this study, $42.0 \%$ of pT1a patients were included in the high-risk group, while approximately half of the pT1b patients were categorized into the low-risk group that had better prognosis.

Previous retrospective studies have evaluated the effect of adjuvant chemotherapy on small nodenegative breast cancer including TNBC and other subtypes.[16-19, 29] Despite some conflicting findings, these studies unanimously recommended adjuvant chemotherapy for pT1c, partially for pT1b, and never for pT1a. Better outcome of adjuvant chemotherapy for small breast cancer seems to be associated with the tumor size. In the current study, pT1b patients had generally higher risks than pT1a patients. However, we also found that nearly half of the low-risk pT1b patients did not benefit from adjuvant chemotherapy. Meanwhile, $42.0 \%$ of the high-risk pT1a patients had a prognostic benefit from chemotherapy.

This study has some limitations. First, both the training and validation sets were from SEER database because we could not obtain appropriately sized external data. Our findings need to be validated in another external data set incorporating more diverse populations, such as younger patients. Second, to ensure the accuracy of the pathological diagnosis of TNBC, the patients diagnosed before 2010 were excluded in the analysis, resulting in a shorter follow-up time and inability of the model to reflect longer prognosis. Fortunately, the risk of recurrence/metastasis has been verified to be significantly lower in TNBC than in other subtypes, 5 years after surgery. Third, other prognostic factors (e.g., Ki-67 index and $\mathrm{LVI}$ ) that may have influenced the treatment decision were not reviewed due to lack the information in the database. Fourth, the data on DFS was not obtained due to database restrictions. Despite these limitations, we were able to evaluate the effect of adjuvant chemotherapy in the largest cohort study to date on PT1abNOMO TNBC. Therefore, our study likely represents the current best available real-world evidence. Other strengths of this study are the detailed clinical and outcome data for BCSS and OS based on nomograms and the STEPP analyses. Our cohort involved 3133 patients with sub-centimetric nodenegative TNBC, which allowed us to perform a stratified analysis and validation test. Further, by adopting the STEPP methodology to investigate absolute chemotherapeutic effect across the nomogram score spectrum, our results will be valuable toward an individualized approach to breast cancer treatment, with the nomogram being helpful for the selection of high-risk patients and the accurate recommendation for adjuvant chemotherapy.

\section{Conclusion}

We constructed and validated nomograms for prognostic prediction in TNBC patients with pT1abN0M0 stage disease. By applying the STEPP methodology, we identified high-risk patients who could potentially benefit from chemotherapy. Our data can be helpful for balancing the risk-benefit ratio, avoiding unnecessary treatment costs, designing future clinical trials, and increasing patient adherence to the prescribed treatment plan.

\section{List Of Abbreviations}


TNBC, triple-negative breast cancer; HER2, human epidermal growth factor receptor 2. SEER, the Surveillance, Epidemiology, and End Results database; OS, overall survival; BCSS, breast cancer-specific survival; PFS, progression-free survival; DMFS, distant-metastasis-free survival; DFS, disease-free survival; STEPP, the subpopulation treatment effect pattern plot; C-index, concordance index; SE, standard error; HR, hazard ratio; LVI, lymphatic vessel invasion; BCS, breast-conserving surgery; CPM, contralateral prophylactic mastectomy; $C T$, chemotherapy; $R A D$, radiotherapy

\section{Declarations}

Ethics approval and consent to participate: This study is approved by the ethics committee of Fudan University Shanghai Cancer Center.

Consent for publication: Not applicable.

Availability of data and materials: The dataset supporting the conclusions of this article is available in the Surveillance, Epidemiology, and End Results (SEER) database, [https://seer.cancer.gov].

Competing interests: The authors declare that there are no competing interests.

Funding: This work was supported by the National Science and Technology Major Project [grant number: 2020ZX09201-013] and National Natural Science Foundation of China [grant number: 81801701].

Authors' contributions: Conception and design: Z.H. Tao, X.C. Hu. Development of methodology: X.Y. Chen, Y.L. Shao, X. Liu. Analysis and interpretation of data: X.Y. Chen, Y.L. Shao, Z.H. Tao, X. Liu. Writing, review and revision of the manuscript: X.Y. Chen, Y.L. Shao, X. Liu, Z.H. Tao, X.C. Hu, J. Zhang, W.X Zhi.

Acknowledgements: We thank Dr Lei Fan from the Department of Breast Surgery for sharing the external validation data, although these were not used in the study due to the small sample size.

\section{References}

1. Garrido-Castro AC, Lin NU, Polyak K. Insights into Molecular Classifications of Triple-Negative Breast Cancer: Improving Patient Selection for Treatment. Cancer Discov. 2019;9(2):176-98.

2. Dent R, Trudeau M, Pritchard KI, Hanna WM, Kahn HK, Sawka CA, Lickley LA, Rawlinson E, Sun P, Narod SA. Triple-negative breast cancer: clinical features and patterns of recurrence. Clin Cancer Res. 2007;13(15 Pt 1):4429-34.

3. Rhee J, Han SW, Oh DY, Kim JH, Im SA, Han W, Park IA, Noh DY, Bang YJ, Kim TY. The clinicopathologic characteristics and prognostic significance of triple-negativity in node-negative breast cancer. BMC Cancer. 2008;8:307.

4. Mustacchi G, De Laurentiis $M$. The role of taxanes in triple-negative breast cancer: literature review. Drug Des Devel Ther. 2015;9:4303-18. 
5. Carey LA, Dees EC, Sawyer L, Gatti L, Moore DT, Collichio F, Ollila DW, Sartor Cl, Graham ML, Perou $\mathrm{CM}$. The triple negative paradox: primary tumor chemosensitivity of breast cancer subtypes. Clin Cancer Res. 2007;13(8):2329-34.

6. Liedtke C, Mazouni C, Hess KR, Andre F, Tordai A, Mejia JA, Symmans WF, Gonzalez-Angulo AM, Hennessy B, Green M, et al. Response to neoadjuvant therapy and long-term survival in patients with triple-negative breast cancer. J Clin Oncol. 2008;26(8):1275-81.

7. Theriault RL, Litton JK, Mittendorf EA, Chen H, Meric-Bernstam F, Chavez-Macgregor M, Morrow PK, Woodward WA, Sahin A, Hortobagyi GN, et al. Age and survival estimates in patients who have nodenegative T1ab breast cancer by breast cancer subtype. Clin Breast Cancer. 2011;11(5):325-31.

8. Hanrahan EO, Gonzalez-Angulo AM, Giordano SH, Rouzier R, Broglio KR, Hortobagyi GN, Valero V. Overall survival and cause-specific mortality of patients with stage T1a,bNOMO breast carcinoma. $\mathrm{J}$ Clin Oncol. 2007;25(31):4952-60.

9. Gradishar WJ, Anderson BO, Abraham J, Aft R, Agnese D, Allison KH, Blair SL, Burstein HJ, Dang C, Elias AD, et al. Breast Cancer, Version 3.2020, NCCN Clinical Practice Guidelines in Oncology. J Natl Compr Canc Netw. 2020;18(4):452-78.

10. Senkus E, Kyriakides S, Ohno S, Penault-Llorca F, Poortmans P, Rutgers E, Zackrisson S, Cardoso F, Committee EG. Primary breast cancer: ESMO Clinical Practice Guidelines for diagnosis, treatment and follow-up. Ann Oncol. 2015;26(Suppl 5):v8-30.

11. Sharma P, Lopez-Tarruella S, Garcia-Saenz JA, Ward C, Connor CS, Gomez HL, Prat A, Moreno F, Jerez-Gilarranz Y, Barnadas A, et al. Efficacy of Neoadjuvant Carboplatin plus Docetaxel in TripleNegative Breast Cancer: Combined Analysis of Two Cohorts. Clin Cancer Res. 2017;23(3):649-57.

12. Sharma P, Lopez-Tarruella S, Garcia-Saenz JA, Khan QJ, Gomez HL, Prat A, Moreno F, Jerez-Gilarranz Y, Barnadas A, Picornell AC, et al. Pathological Response and Survival in Triple-Negative Breast Cancer Following Neoadjuvant Carboplatin plus Docetaxel. Clin Cancer Res. 2018;24(23):5820-9.

13. Loibl S, O'Shaughnessy J, Untch M, Sikov WM, Rugo HS, McKee MD, Huober J, Golshan M, von Minckwitz G, Maag D, et al. Addition of the PARP inhibitor veliparib plus carboplatin or carboplatin alone to standard neoadjuvant chemotherapy in triple-negative breast cancer (BrighTNess): a randomised, phase 3 trial. Lancet Oncol. 2018;19(4):497-509.

14. Sparano JA, Wang M, Martino S, Jones V, Perez EA, Saphner T, Wolff AC, Sledge GW Jr, Wood WC, Davidson NE. Weekly paclitaxel in the adjuvant treatment of breast cancer. N Engl J Med. 2008;358(16):1663-71.

15. Jones S, Holmes FA, O'Shaughnessy J, Blum JL, Vukelja SJ, Mclntyre KJ, Pippen JE, Bordelon JH, Kirby RL, Sandbach J, et al. Docetaxel With Cyclophosphamide Is Associated With an Overall Survival Benefit Compared With Doxorubicin and Cyclophosphamide: 7-Year Follow-Up of US Oncology Research Trial 9735. J Clin Oncol. 2009;27(8):1177-83.

16. Vaz-Luis I, Ottesen RA, Hughes ME, Mamet R, Burstein HJ, Edge SB, Gonzalez-Angulo AM, Moy B, Rugo HS, Theriault RL, et al. Outcomes by tumor subtype and treatment pattern in women with small, node-negative breast cancer: a multi-institutional study. J Clin Oncol. 2014;32(20):2142-50. 
17. de Nonneville A, Goncalves A, Zemmour C, Cohen M, Classe JM, Reyal F, Colombo PE, Jouve E, Giard $\mathrm{S}$, Barranger $\mathrm{E}$, et al. Adjuvant chemotherapy in pT1ab node-negative triple-negative breast carcinomas: Results of a national multi-institutional retrospective study. Eur J Cancer. 2017;84:3443.

18. Ren YX, Hao S, Jin X, Ye FG, Gong Y, Jiang YZ, Shao ZM. Effects of adjuvant chemotherapy in T1N0M0 triple-negative breast cancer. Breast. 2019;43:97-104.

19. Steenbruggen TG, van Werkhoven E, van Ramshorst MS, Dezentje VO, Kok M, Linn SC, Siesling S, Sonke GS. Adjuvant chemotherapy in small node-negative triple-negative breast cancer. Eur J Cancer. 2020;135:66-74.

20. Wolbers M, Koller MT, Witteman JC, Steyerberg EW. Prognostic models with competing risks: methods and application to coronary risk prediction. Epidemiology. 2009;20(4):555-61.

21. Bonetti M, Gelber RD. Patterns of treatment effects in subsets of patients in clinical trials. Biostatistics. 2004;5(3):465-81.

22. Bonetti $\mathrm{M}$, Gelber RD. A graphical method to assess treatment-covariate interactions using the Cox model on subsets of the data. Stat Med. 2000;19(19):2595-609.

23. Lazar AA, Cole BF, Bonetti M, Gelber RD. Evaluation of treatment-effect heterogeneity using biomarkers measured on a continuous scale: subpopulation treatment effect pattern plot. J Clin Oncol. 2010;28(29):4539-44.

24. Frank E, Harrell. J. rms: Regression Modeling Strategies. R Package version 5.1-1. http://CRAN.Rproject.org/package $=$ rms.

25. Sparano JA, Gray RJ, Makower DF, Pritchard KI, Albain KS, Hayes DF, Geyer CE Jr, Dees EC, Perez EA, Olson JA Jr, et al. Prospective Validation of a 21-Gene Expression Assay in Breast Cancer. N Engl J Med. 2015;373(21):2005-14.

26. Cardoso F, van't Veer LJ, Bogaerts J, Slaets L, Viale G, Delaloge S, Pierga JY, Brain E, Causeret S, DeLorenzi M, et al. 70-Gene Signature as an Aid to Treatment Decisions in Early-Stage Breast Cancer. N Engl J Med. 2016;375(8):717-29.

27. Engel J, Eckel R, Kerr J, Schmidt M, Furstenberger G, Richter R, Sauer H, Senn HJ, Holzel D. The process of metastasisation for breast cancer. Eur J Cancer. 2003;39(12):1794-806.

28. Husemann Y, Geigl JB, Schubert F, Musiani P, Meyer M, Burghart E, Forni G, Eils R, Fehm T, Riethmuller G, et al. Systemic spread is an early step in breast cancer. Cancer Cell. 2008;13(1):58-68.

29. Gamucci T, Vaccaro A, Ciancola F, Pizzuti L, Sperduti I, Moscetti L, Longo F, Fabbri MA, Giampaolo MA, Mentuccia L, et al. Recurrence risk in small, node-negative, early breast cancer: a multicenter retrospective analysis. J Cancer Res Clin Oncol. 2013;139(5):853-60.

\section{Figures}




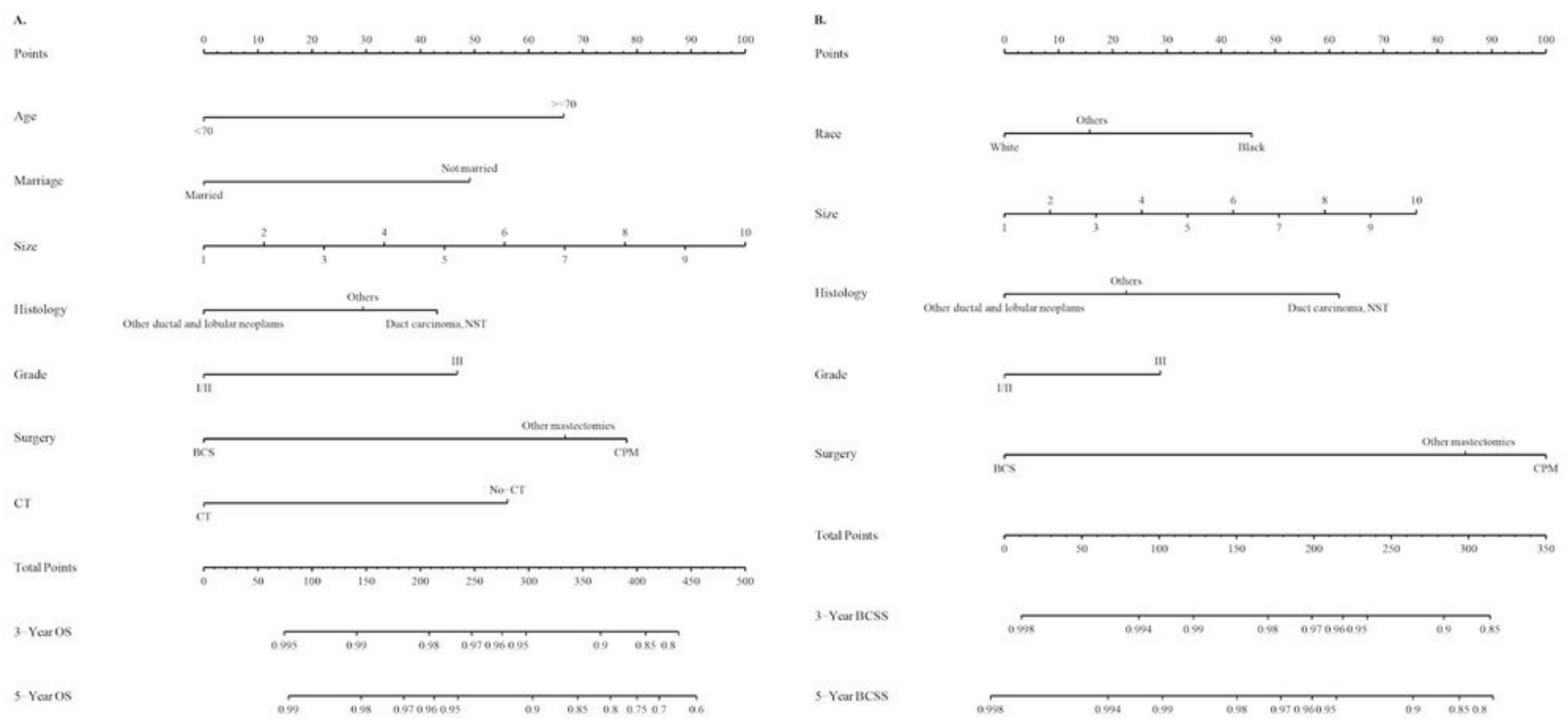

\section{Figure 1}

Nomograms for predicting 3- and 5-year survival probabilities in T1abNOMO TNBC. (A) OS, (B) BCSS. We drew a vertical line from the variable value to the "Points" axis to determine the point for each variable. After adding up all points, the total points are added to the bottom scales to identify the 3- and 5-year survival probabilities. OS, Overall survival; BCSS, breast cancer-specific survival; NST, no special type; $\mathrm{BCS}$, breast-conserving surgery; CPM, contralateral prophylactic mastectomy; $\mathrm{CT}$, chemotherapy 

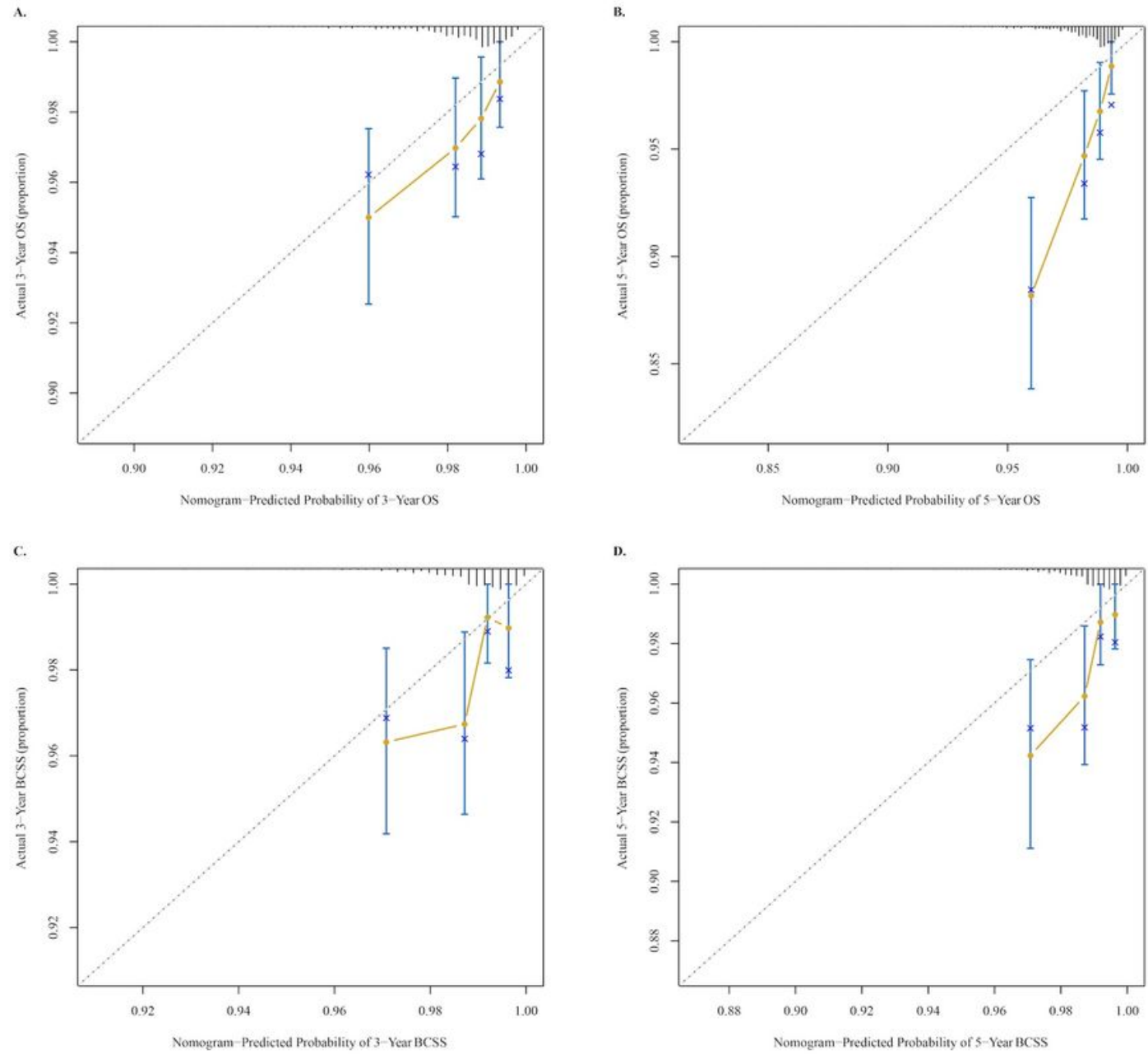

\section{Figure 2}

Calibration curves for predicting survival in the training cohort. The (A) 3-year and (B) 5-year OS and the (C) 3-year and (D) 5-year BCSS. Nomogram-predicted probability of survival is plotted on the x-axis, while actual survival is on the y-axis. Vertical bars indicate $95 \%$ Cls measured using the Kaplan-Meier method. Orange dots indicate the mean predicted value. The dashed 45-degree diagonal lines through the origin point represent the perfect calibration model. OS, overall survival; BCSS, breast cancer-specific survival; $\mathrm{Cl}$, confidence interval 
A.
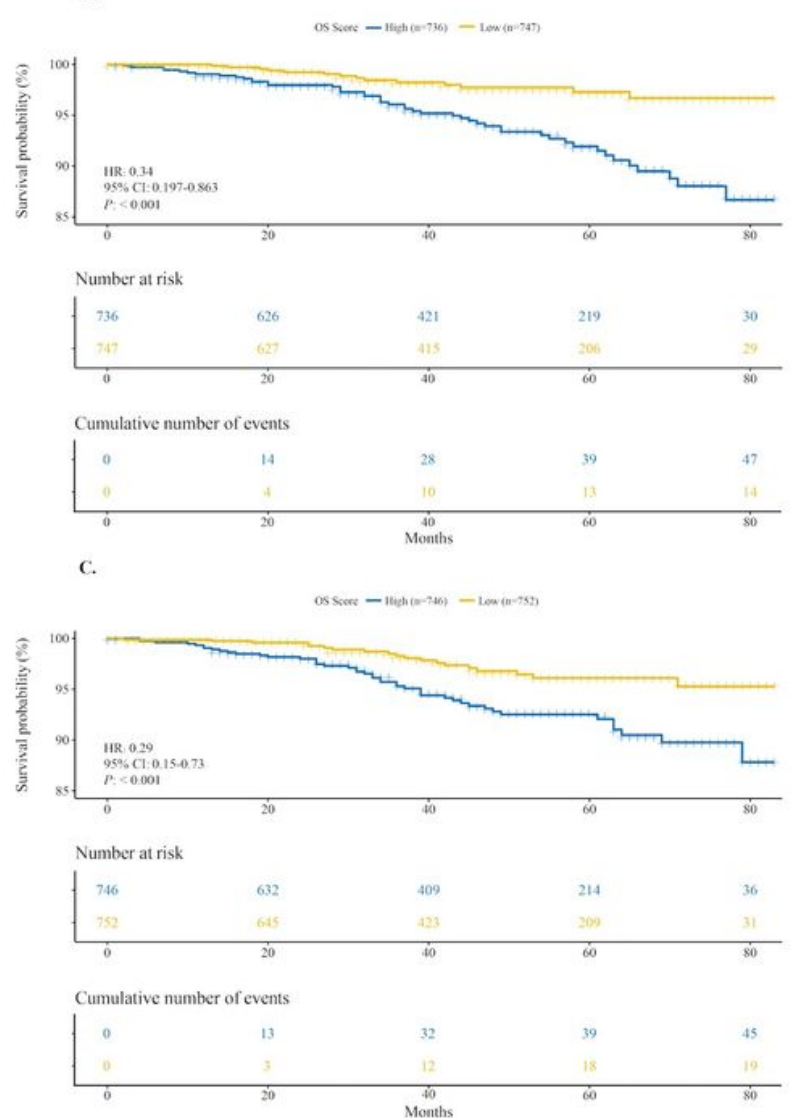
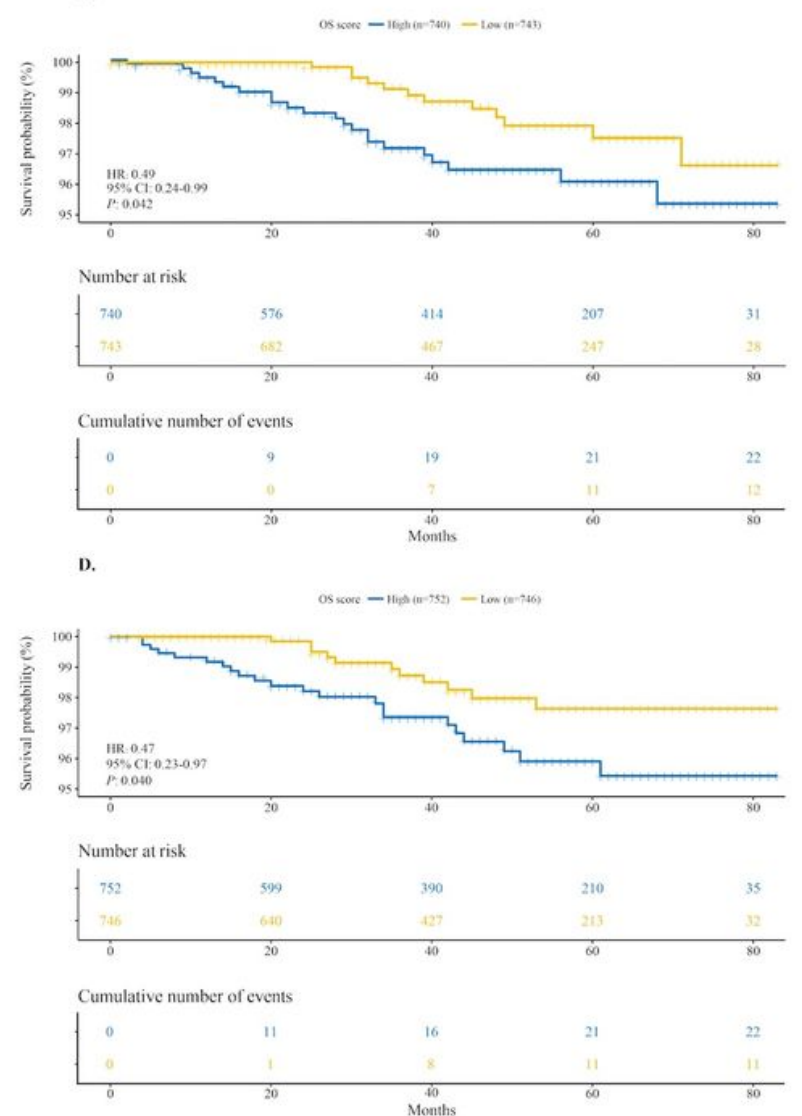

\section{Figure 3}

Comparison of OS by nomogram risk stratification. The (A) OS and (B) BCSS of the training cohort and the (C) OS and (D) BCSS of the validation cohort. OS, overall survival; BCSS, breast cancer-specific survival 
A.

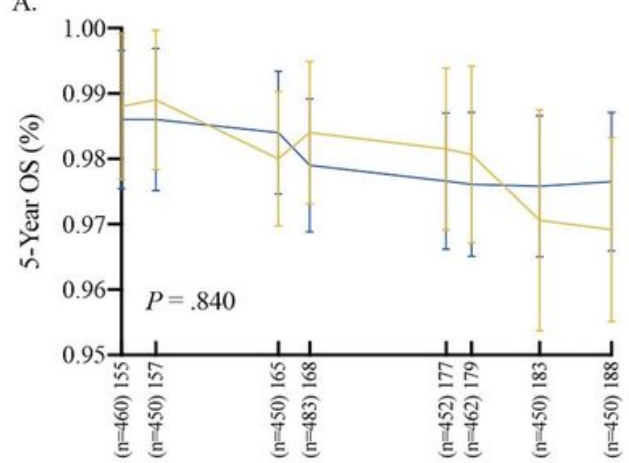

Median OS Score in Subpopulation

C.

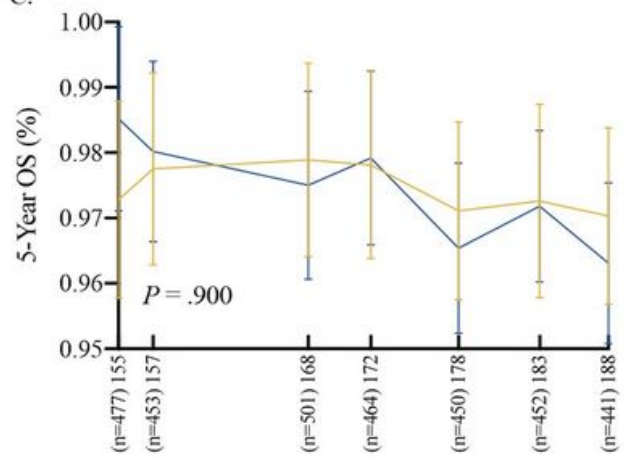

Median OS Score in Subpopulation
- No-CT $(\mathrm{n}=267)$

- CT $(\mathrm{n}=480)$

- No-CT $(\mathrm{n}=257)$

- CT (n=495)
B.

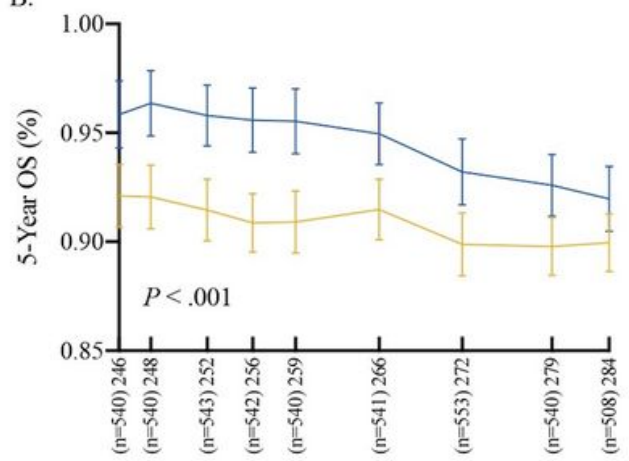

Median OS Score in Subpopulation

D.

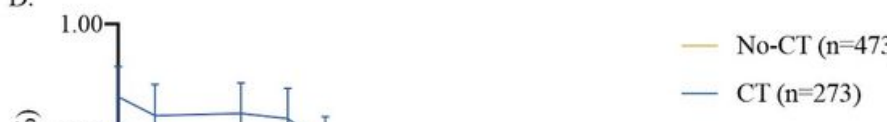

Figure 4

STEPP of 5-year OS defined by chemotherapy. The (A) low-risk and (B) high-risk patients in the training cohort and the (C) low-risk and (D) high-risk patients in the validation cohort. STEPP, the subpopulation treatment effect pattern plot; OS, overall survival; CT, chemotherapy. 

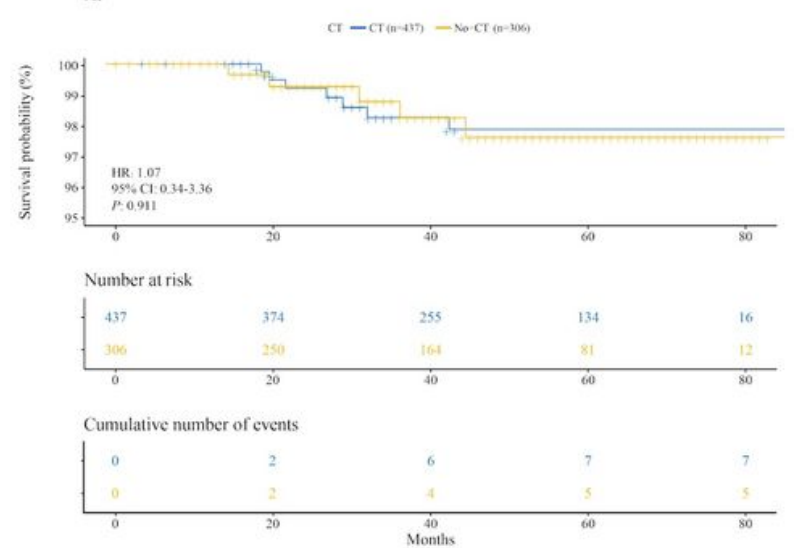

C.
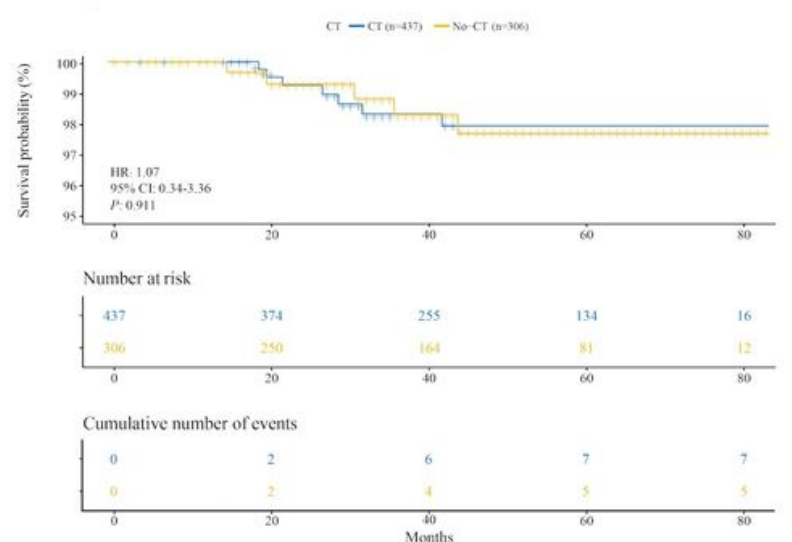

B.
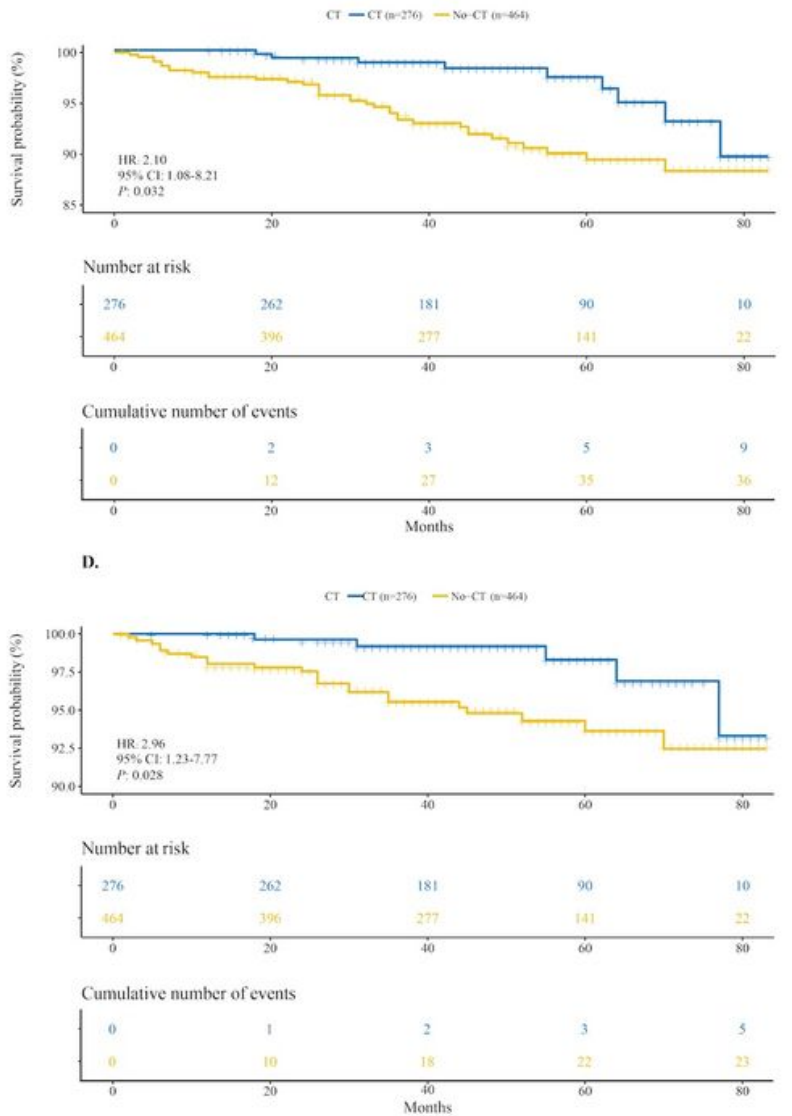

\section{Figure 5}

Comparison of survival by chemotherapy status in the training cohort. OS of the (A) low-risk and (B) highrisk patients and the BCSS of the (C) low-risk and (D) high-risk patients. OS, overall survival; BCSS, breast cancer-specific survival; $\mathrm{CT}$, chemotherapy 
A.
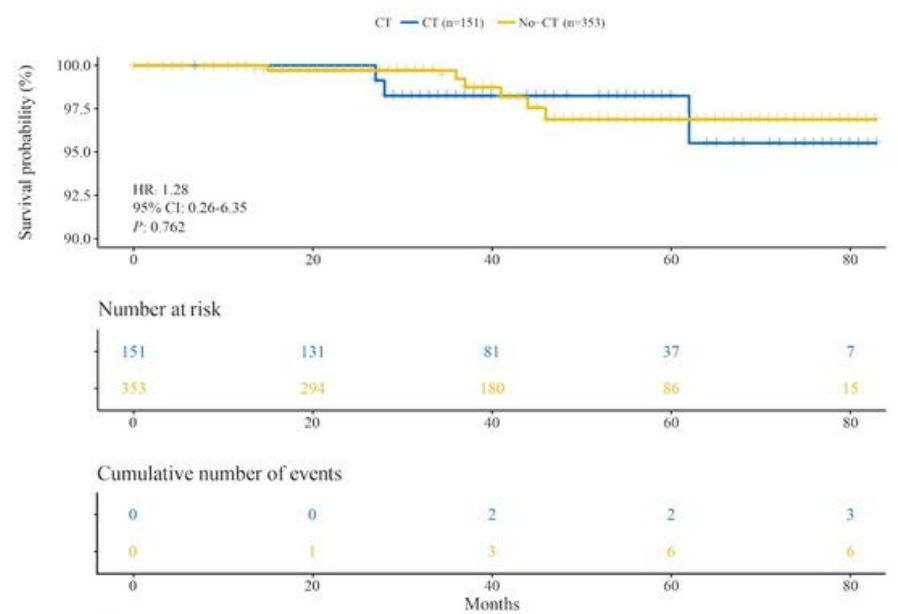

c.
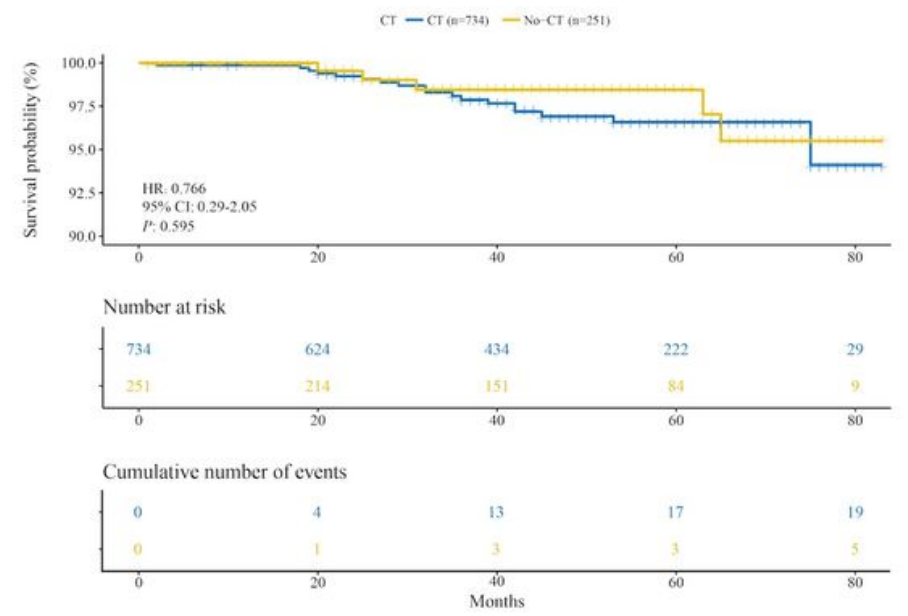

B.
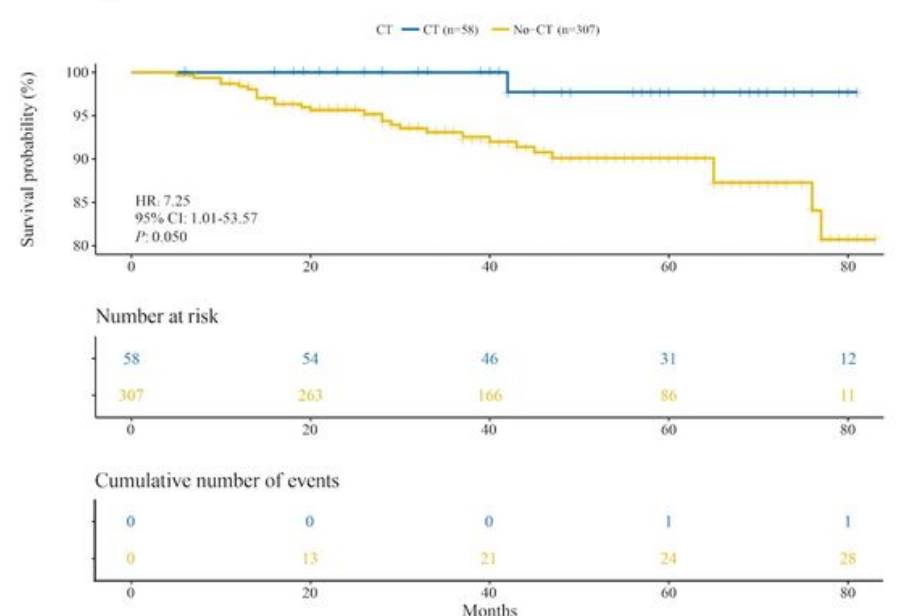

D.
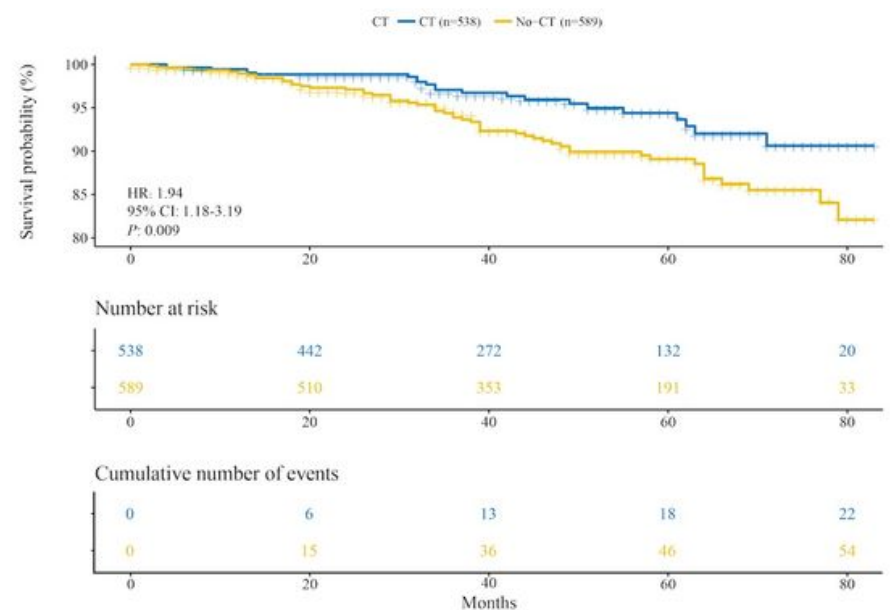

Figure 6

Comparison of OS by chemotherapy status in the nomogram score subgroups of $\mathrm{pT} 1 \mathrm{a} / \mathrm{b}$. The (A) lowrisk and (B) high-risk pT1a patients and the (C) low-risk and (D) high-risk pT1b patients. OS, overall survival; $\mathrm{CT}$, chemotherapy

\section{Supplementary Files}

This is a list of supplementary files associated with this preprint. Click to download.

- Supplementalfigure1.tiff

- Supplementalfigure2.tiff

- Supplementalfigure3.tiff

- Supplementalfigure4.tiff

- Supplementaltables.docx 